\title{
Synthesis, Characterization and Cytotoxicity Evaluation of New Compounds from 0xazol-5(4H)-ones and Oxazoles Class Containing 4-(4-Bromophenylsulfonyl)phenyl Moiety
}

\author{
THEO DORA-VENERA APO STOL ${ }^{1}$, STEFANIA-FELICIA BARBUCEANU1*, OCTAVIAN TUDOREL OLARU ${ }^{2}$, CONSTANTIN DRAGHICI ${ }^{3}$, \\ GABRIEL SARAMET ${ }^{4}$, BO GDAN SOCEA ${ }^{5}$, CRISTIAN ENACHE ${ }^{6}$, LAURA-ILEANA SOCEA ${ }^{1}$ \\ ${ }^{1}$ Carol Davila University of Medicine and Pharmacy, Faculty of Pharmacy, Organic Chemistry Department, 6 Traian Vuia Str., \\ 020956, Bucharest, Romania \\ ${ }^{2}$ Carol Davila University of Medicine and Pharmacy, Faculty of Pharmacy, Pharmaceutical Botany and Cell Biology Department, \\ 6 Traian Vuia Str., 020956, Bucharest, Romania \\ ${ }^{3}$ Military Medical Scientific Research Center, 24-28 Gr. Cobalcescu Str., 010195, Bucharest, Romania \\ ${ }^{4}$ Carol Davila University of Medicine and Pharmacy, Faculty of Pharmacy, Pharmaceutical Technologyand Biopharmacy Department, \\ 6 Traian Vuia Str., 020956, Bucharest, Romania \\ ${ }^{5}$ Carol Davila University of Medicine and Pharmacy, Faculty of General Medicine, St. Pantelimon Emergency Hospital, 340-342 \\ Pantelimon Road, 021659, Bucharest, Romania \\ ${ }^{6}$ Central Laboratory for Phytosanitary Quarantine, 11 Afumati Road, 077190, Bucharest, Romania
}

\begin{abstract}
Steiger $N$-acylation of $\alpha$-alanine with 4-(4-bromophenylsulfonyl)benzoyl chloride led to 2-[4-(4bromophenylsulfonyl) benzamido] propanoic acid. This compound underwent intramolecular cyclization in the presence of $\mathrm{N}$-methylmorpholine and ethyl chloroformate or acetic anhydride to the corresponding saturated azlactone. Then acylaminoacylation of dry aromatic hydrocarbons with 2-[4-(4bromophenylsulfonyl)phenyl]-4-methyloxazol-5(4H)-one or 2-[4-(4-bromophenylsulfonyl) benzamido Jpropanoyl chloride in the presence of anhydrous aluminum chloride led to corresponding $\alpha$-acylamino ketones. These new intermediates were heterocyclized under the action of phosphorus oxychloride or concentrated sulfuric acid in the presence of acetic anhydride to the corresponding oxazoles. The newly synthesized compounds were characterized by spectral studies (FT-IR, UV-Vis, MS, ${ }^{1} \mathrm{H}$ - and ${ }^{13} \mathrm{C}-N M R$ ) and elemental analysis. The purity of the new compounds was evaluated by RP-HPLC. The experimental research regarding to the in vitro cytotoxic activity of the new compounds were performed using Daphnia magna bioassay.
\end{abstract}

Keywords: $N$-acyl- $\alpha$-amino acid, 1,3-oxazol-5(4H)-one, $\alpha$-acylamino ketone, 1,3-oxazole, cytotoxicity

Heterocyclic compounds containing 1,3-oxazol-5(4H)one and 1,3-oxazole ring are important targets in synthetic and medicinal chemistry, because of their applications as active substances.

1,3-0xazoles are substructures of various biologically active natural products, pharmaceuticals, and synthetic intermediates. Thus, the 1,3-oxazole nucleus is an important pharmacophore in modern drugs, due to having a wide spectrum of biological activities [1], such as antiinflammatory (e.g. Oxaprozin, Romazarit, Ditazol, Isamoxole, Tioxaprofene, Tilmacoxib) [2], analgesic (e.g. Oxaprozin) [3], antibacterial, antifungal (e.g. Sulfamoxole, Sulfaguanole) [4], anti-diabetic (e.g. Aleglitazar, Farglitazar, Darglitazone, Muraglitazar, Imiglitazar) [5], antitumoral (Mubritinib) [6], anti-tuberculosis [7], muscle relaxant (e.g. Azumolene) [8], antioxidant [9], and HIV-inhibitor effect [10]. Moreover, various natural products of peptide origin containing oxazole ring are active substances which exhibit several pharmacological properties [11], including antitumoral (e.g. Telomestatin, Thiangazole, Diazonamide A, Mycalolide A, Leucascandrolide A), analgesic (e.g. Hennoxazole A), antifungal (e.g. Rhizoxin D, Phorboxazole A and B, Leucascandrolide A, Bengazole A, Mycalolide A), antibacterial (e.g. Pristinamycin IIB or Virginiamicin $M_{2}$, Bistratamide, Sulfomycin I, Griseoviridin, Madumycin If', Oxazolomycin, Microcin B17, Promothiocin A, Flopristin), antiviral (e.g. Hennoxazole A, Thiangazole, Phenoxan), antimycobacterial (e.g. Texaline), antileukemia (e.g. Ulapualide A), and anticonvulsant (e.g. Pimprinine).

The interest in the chemistry of the saturated azlactones - which are internal anhydrides of acyl amino acids - is due to their usefulness as intermediate in the synthesis of different heterocyclic compounds or modified á-amino acids or their derivatives [12]. Also, 1,3-oxazol-5(4H)-ones have been reported to present antimicrobial [13], antitumoral [14], antiviral activities [15] etc.

Further, diaryl sulfone derivatives (e.g. Dapsone, Amidapsone, Acedapsone, Promanide, Solasulfone, Sulfoxone, Diuciphone) were also found to possess antibacterial, antiviral, anti-tuberculosis, and antioxidant action [16]. In the view of these reports, the diaryl sulfone moiety was incorporated into various heterocyclic systems with potential biological activity [17-19].

Based on all above considerations and also in continuation of our researches $[20,21]$, in this work is reported the synthesis and characterization of new heterocyclic compounds from oxazol-5(4H)-ones and oxazoles class wherein the 2-aryl group is 4-(4bromophenylsulfonyl)phenyl and of their acyclic intermediates, with the aim to obtain potent biologically active compounds. The synthetized compounds were tested for cytotoxic activity using Daphnia magna bioassay. The method is simple, rapid, and can predict the biological effect [22-26].

\footnotetext{
*email: sbarbuceanu@gmail.com; stefaniafelicia_barbuceanu@yahoo.com; Phone: 0722763428
} 


\section{Experimental part}

Chemistry

Melting points were determined on a Böetius apparatus and are uncorrected. FT-IR spectra were recorded in $\mathrm{KBr}$ pellets on a Bruker Vertex 70 spectrometer; intensity of IR bands are given as: weak (w), medium (m), strong (s), and very strong (vs). UV-Vis spectra were registered in methanolic solution $\left(2 \cdot 5.10^{-5} \mathrm{M}\right)$ on an Analytik Jena AG Specord 40 spectrophotometer. NMR spectra were recorded on a Varian Gemini 300BB spectrometer at 300 $\mathrm{MHz}$ for ${ }^{1 \mathrm{H}-N M R}$ and $75 \mathrm{MHz}$ for ${ }^{13} \mathrm{C}-\mathrm{NMR}$ using DMSO-d $\mathrm{d}_{6}$ or $\mathrm{CDCl}_{3}$ as solvents; chemical shifts $(\delta)$ are reported in ppm relative to tetramethylsilane (TMS) as internal standard and coupling constants $(J)$ are expressed in $\mathrm{Hz}$. For multiplicity of signals in ${ }^{1} \mathrm{H}$-NMR spectra, following abbreviations were used: singlet (s), broad singlet (br s), doublet (d), broad doublet ( $b r d)$, doublet of doublets (dd), triplet (t), broad triplet (brt), triplet of triplets ( $(\mathrm{t})$, quartet (q), quintet (quint), and multiplet $(\mathrm{m})$. Mass spectra (ESI-MS/MS) were recorded on a Varian 1200 LC-MS/MS high performance liquid chromatograph coupled with a triple quadrupole mass spectrometer with electrospray interface (ESI), by positive and/or negative ionization. GC-El-MS analysis was carried out using a Fisons Instruments GC 8000 with electron impact quadrupole and MD 800 mass spectrometer detector. Compounds purity was checked by RP-HPLC using a Beckman System Gold 126 liquid chromatograph, equipped with a System Gold 166 UV-Vis detector; retention time $\left(t_{R}\right)$ of compounds in min is reported. Contents of $C, H, N$, and $S$ were determined using a Costech ECS 4010 micro elemental analyzer.

\section{Synthesis and characterization of compounds}

The synthetic method used in this approach consisted in $\mathrm{N}$-acylation of $\alpha$-alanine with benzoyl chloride 2 by Steiger procedure to the $N$-acyl- $\alpha$-alanine 3 , followed by cyclization of this compound to the corresponding saturated azlactone 4. Friedel-Crafts acylaminoacylation of aromatic hydrocarbons (benzene, toluene, $m$-xylene, mesytilene) with 1,3-oxazol-5(4H)-one (2-oxazolin-5-one) 4 or $N$-acyl- $\alpha$-alanyl chloride 5 , in the presence of anhydrous aluminum chloride, yielded the corresponding $\alpha$-acylamino ketones $6 \mathbf{a}-\mathbf{d}$. These intermediates were converted into 1,3-oxazoles 7a-d by Robinson-Gabriel cyclodehydration with phosphorus oxychloride or concentrated sulfuric acid in the presence of acetic anhydride (scheme 1). The structures of new synthesized compounds 3-7 were established unequivocally by FT-IR, UV-Vis, MS, ${ }^{1} \mathrm{H}-\mathrm{NMR}$, ${ }^{13} \mathrm{C}-\mathrm{NMR}$ spectra and elemental analysis.

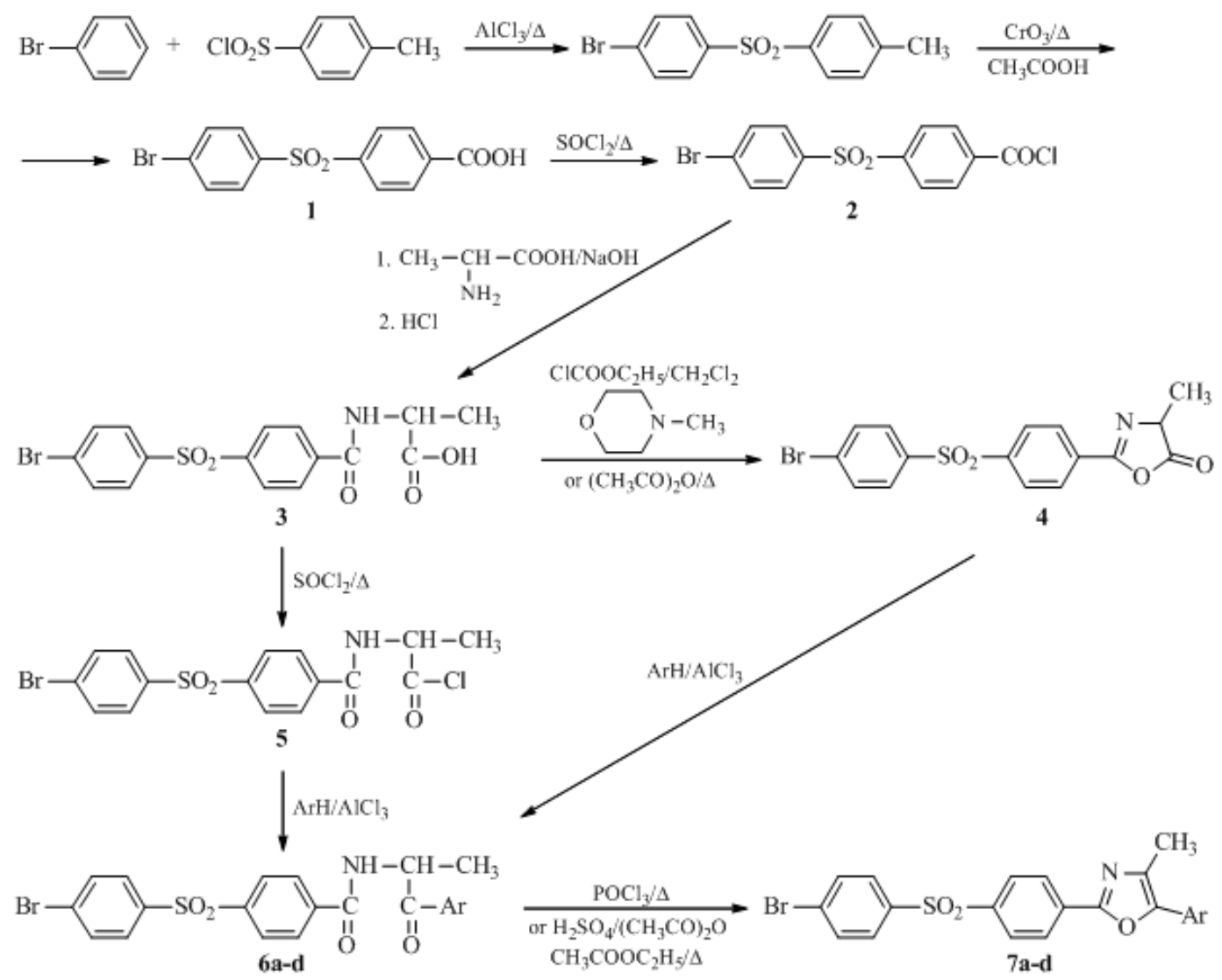

6a, $7 \mathbf{a} \mathrm{Ar}=\mathrm{C}_{6} \mathrm{H}_{5}$

6b, 7b $\mathrm{Ar}=4-\mathrm{CH}_{3}-\mathrm{C}_{6} \mathrm{H}_{4}$

6c, 7c $\mathrm{Ar}=2,4-\left(\mathrm{CH}_{3}\right)_{2} \mathrm{C}_{6} \mathrm{H}_{3}$

6d, 7d $\mathrm{Ar}=2,4,6-\left(\mathrm{CH}_{3}\right)_{3} \mathrm{C}_{6} \mathrm{H}_{2}$

Scheme 1. Synthesis of the compounds

\section{Synthesis of 2-[4-(4-bromophenylsulfonyl)benzamido]propanoic acid $\mathbf{3}$}<smiles>C[C](C)C(=O)NC(=O)c1ccc(OC(=O)c2ccc(Br)cc2)cc1</smiles>

Fig. 1. The structure of compound 3 with atom numbering (for NMR assignments) 
$\alpha$-Alanine $(1.78 \mathrm{~g}, 20 \mathrm{mmol}$ ) was dissolved in $20 \mathrm{~mL}$ of $1 \mathrm{~N} \mathrm{NaOH}$ solution. This solution was cooled to $0-5^{\circ} \mathrm{C}$ and then two solutions were added simultaneously dropwise under continuous stirring for 30 min, as follows: a solution of crude acyl chloride 2 (7.19 g, $20 \mathrm{mmol}$ ) in $45 \mathrm{~mL}$ anhydrous $\mathrm{CH}_{2} \mathrm{Cl}_{2}$ and $10 \mathrm{~mL}$ of $2 \mathrm{~N} \mathrm{NaOH}$ solution, respectively. After $1 \mathrm{~h}$ stirring at room temperature, the aqueous layer was separated and then acidified with $2 \mathrm{~N}$ $\mathrm{HCl}$. The precipitate was filtered off and recrystallized from water as white needle-shaped crystals; yield 96\%; m.p. 197-198 ${ }^{\circ} \mathrm{C}$;

UV-Vis ( $\left.\mathrm{CH}_{3} \mathrm{OH}, \lambda \mathrm{nm}\right)(\mathrm{lg} \varepsilon): 202.6$ (4.46), 226.4 (4.08), 252.0 (4.36);

FT-IR $\left(\mathrm{KBr}, v \mathrm{~cm}^{-1}\right): 3377 \mathrm{~s}, 3088 \mathrm{~m}, 3066 \mathrm{~m}, 2998 \mathrm{~m}$, $2952 \mathrm{~m}, 2872 \mathrm{w}, 1708 \mathrm{vs}, 1644 \mathrm{vs}, 1577 \mathrm{~s}, 1487 \mathrm{~s}, 1462 \mathrm{~m}$, 1523vs, 1323vs, 1296vs, 1158vs, 853s, 571vs;

${ }^{1} \mathrm{H}-N M R\left(D M S O-\mathrm{d}_{1}, \delta \mathrm{ppm}, J \mathrm{~Hz}\right): 1.38(\mathrm{~d}, 7.3,3 \mathrm{H}, \mathrm{H}-18)$, 4.41 (quint, 7.3, 1H, $\left.\mathrm{H}^{\prime}-4\right), 7.85$ (d, 8.8, 2H, H-14, H-16), 7.92 (d, 8.8, 2H, H-13, H-17), 8.06 (d, 8.5, 2H, H-7, H-11), 8.09 (d, $8.5,2 \mathrm{H}, \mathrm{H}-8, \mathrm{H}-10), 8.95(\mathrm{~d}, 7.3,1 \mathrm{H}, \mathrm{NH})$;

${ }^{13} \mathrm{C}-\mathrm{NMR}$ (DMSO-d $\mathrm{d}_{6} \delta \mathrm{ppm}$ ): 16.79 (C-18), 48.36 (C-4), 127.65 (C-8, C-10), 128.28 (C-15), 128.89 (C-7, C-11), 129.52 (C-13, C-17), 133.00 (C-14, C-16), 138.73 (C-6), 139.92 (C-12), 142.78 (C-9), 164.88 (C-2), 173.91 (C-5);

+ ESI-MS/MS (m/z, rel. abund. \%): $412\left({ }^{79} \mathrm{Br}\right) / 414\left({ }^{81} \mathrm{Br}\right)$ $[\mathrm{M}+\mathrm{H}]^{+} ; 394 / 396(19.8 / 17.6)\left[\mathrm{M}+\mathrm{H}-\mathrm{H}_{2} \mathrm{O}\right]^{+} ; 366 / 368$ (66.2/ $67.3)\left[\mathrm{M}+\mathrm{H}-\mathrm{H}_{2} \mathrm{O}-\mathrm{CO}\right]^{+} ; 323 / 325$ (100, BP) $\left[\mathrm{BrC}_{6} \mathrm{H}_{4} \mathrm{SO}_{2}\right.$ $\mathrm{C}_{6} \mathrm{H}_{4} \mathrm{CO}^{+} ; 203 / 205\left[\mathrm{BrC}_{6} \mathrm{H}_{5} \mathrm{SO}\right]^{+} ;$

-ESI-MS/MS (m/z, rel. abund. \%): $410\left({ }^{79} \mathrm{Br}\right) / 412\left({ }^{81} \mathrm{Br}\right)$ [M-H]; 366/368 (23.8/24.2) [M-H-CO ] ; 338/340 (12.8/ 13.6) $\left[\mathrm{BrC}_{6} \mathrm{H}_{4} \mathrm{SO}_{2} \mathrm{C}_{6} \mathrm{H}_{4} \mathrm{CONH}\right] ; 2295 / 297$ (100, BP) [ $\left.\mathrm{BrC}_{6} \mathrm{HO}_{2} \mathrm{C}_{6} \mathrm{H}^{4}\right] ; ; 219 / 221\left[\mathrm{BrCH}^{4} \mathrm{SO}_{2}\right] ;$

$\mathrm{RP}^{4}-\mathrm{HPLC}\left(\mathrm{CH}_{3} \mathrm{OH}: \mathrm{H}_{2} \mathrm{O}=30: 70,1^{2} \mathrm{~mL} / \mathrm{min}, 250 \mathrm{~nm}\right)$ : purity $99.99 \% ; t_{8}^{3} .53 \mathrm{~min}$;

Anal. (\%): Calcd. for $\mathrm{C}_{1} \mathrm{H}_{13} \mathrm{BrNO}_{5} \mathrm{~S}(412.26 \mathrm{~g} / \mathrm{mol})$ : $\mathrm{C}$, 46.61; H, 3.42; N, 3.40; S, 7.78. Found: C, 46.68; H, 3.40; N, 3.45; S, 7.76 .

Synthesis of 2-[4-(4-bromophenylsulfonyl)phenyl]-4methyloxazol-5(4H)-one 4

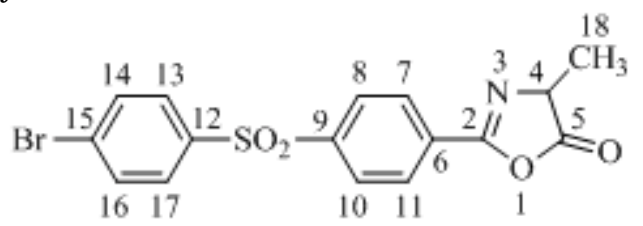

Fig. 2. The structure of compound 4 with atom numbering (for NMR assignments)

Method 1. 2-[4-(4-Bromophenyl sulfonyl) benzamido] propanoic acid $3(4.33 \mathrm{~g}, 10.5 \mathrm{mmol})$ and $\mathrm{N}$-methylmorpholine $(1.15 \mathrm{~mL}, 10.5 \mathrm{mmol})$ were added under stirring into $50 \mathrm{~mL} \mathrm{CH} \mathrm{Cl}_{\text {at }}$ at room temperature. An equimolar quantity of ethyl chloroformate $(1 \mathrm{~mL})$ was then added dropwise to the reaction mass. The mixture was magnetically stirred for 30 min at ambient temperature and then poured over $100 \mathrm{~mL}$ cold water. The organic layer was separated and washed with $5 \% \mathrm{NaHCO}_{3}$ solution and then with water. After drying over $\mathrm{MgSO}_{4}$, the solvent was removed under reduced pressure. The solid product was purified by recrystallization from cyclohexane as white crystals; $97 \%$ yield.

Method 2. 2-[4-(4-Bromophenylsulfonyl) benzamido] propanoic acid $\mathbf{3}(2.06 \mathrm{~g}, 5 \mathrm{mmol})$ in an 8-fold molar excess of acetic anhydride $(3.8 \mathrm{~mL}, 40 \mathrm{mmol})$ was heated under stirring at $140^{\circ} \mathrm{C}$ for about $1 \mathrm{~h}$ until all crystals have dissolved. Reaction mixture was then heated for another 30 min with constant stirring, until azlactone was crystallized. After cooling at room temperature, the precipitate was filtered off and washed on the filter with a small amount of cool ethanol. The product was obtained as white crystals; $98 \%$ yield; m.p. $162-164^{\circ} \mathrm{C}$ (cyclohexane);

UV-Vis $\left(\mathrm{CH}_{3} \mathrm{OH}, \lambda \mathrm{nm}\right)(\mathrm{lg} \varepsilon): 202.6$ (4.47), 228.2 (4.08), 252.9 (4.36);

FT-IR $\left(\mathrm{KBr}, v \mathrm{~cm}^{-1}\right): 3092 \mathrm{~m}, 3069 \mathrm{~m}, 2989 \mathrm{~m}, 2943 \mathrm{~m}$, 2874w, 1820vs, 1650vs, 1597m, 1573s, 1472m, 1331vs, 1303s, 1295s, 1256s, 1163vs, 1046s, 846s, 574s;

${ }^{1} \mathrm{H}-\mathrm{NMR}\left(\mathrm{CDCl}{ }_{3}, \delta \mathrm{ppm}, J \mathrm{~Hz}\right): 1.59(\mathrm{~d}, 7.4,3 \mathrm{H}, \mathrm{H}-18)$, 4.49 (q, 7.4, $1 \mathrm{H}, \mathrm{H}-4), 7.68$ (d, 8.8, 2H, H-14, H-16), 7.83 (d, $8.8,2 \mathrm{H}, \mathrm{H}-13, \mathrm{H}-17), 8.05$ (d, 8.5, 2H, H-7, H-11), 8.14 (d, 8.5, $2 \mathrm{H}, \mathrm{H}-8, \mathrm{H}-10)$;

${ }^{13} \mathrm{C}-\mathrm{NMR}\left(\mathrm{CDCl}{ }_{3^{\prime}} \delta \mathrm{ppm}\right): 16.86$ (C-18), 61.39 (C-4), $128.98(C-8, C-10), 129.49$ (C-7, C-11, C-13, C-17), 130.60 (C-6), 131.54 (C-15), 132.97 (C-14, C-16), 139.92 (C-12), 145.03 (C-9), 160.28 (C-2), 178.09 (C-5);

+ ESI-MS/MS (m/z, rel. abund. \%): $394\left({ }^{79} \mathrm{Br}\right) / 396\left({ }^{81} \mathrm{Br}\right)$ [M+H]+; 366/368 (87.6/63.2) [M+H-CO]+; 323/325 (100, BP) [ $\left.\mathrm{BrC}_{6} \mathrm{H}_{4} \mathrm{SO}_{2} \mathrm{C}_{6} \mathrm{H}_{4} \mathrm{CO}\right]^{+} ; 203 / 205 \quad(13.2 / 12.6)$ $\left[\mathrm{BrC}_{6} \mathrm{H}_{4} \mathrm{SO}\right]^{+6}$

GC'-El-MS (m/z, rel. abund. \%): $393\left({ }^{79} \mathrm{Br}\right) / 395\left({ }^{81} \mathrm{Br}\right)(13.1 /$ 19.3) [M]+x; 349/351 (27.7/28.4) [M-CO ] ${ }^{++} ; 323 / 325$ (88.1/100, BP) [M-CO $\left.-\mathrm{C}_{2} \mathrm{H}_{2}\right]+x_{;} ; 203 / 205(40.5 / 50.6)$ [ $\left.\mathrm{BrC}_{6} \mathrm{H}_{5} \mathrm{SO}\right]^{+} ; 155 / 157(25.2 / 28.4)^{\prime}\left[\mathrm{BrC}_{6} \mathrm{H}_{4}\right]^{+} ; 104(25.2)$ $\left[\mathrm{C}_{6} \mathrm{H}_{4} \mathrm{CHNH}^{4} ; 76(31.6)\left[\mathrm{C}_{6} \mathrm{H}_{4}\right]^{+*} ; 50(8.7)^{6}\left[\mathrm{C}_{4} \mathrm{H}_{2}\right]^{++} ; \mathrm{t}_{\mathrm{R}} 30.62\right.$ min;

RP-HPLC ( $\left.\mathrm{CH}_{3} \mathrm{OH}: \mathrm{H}_{2} \mathrm{O}=60: 40,1 \mathrm{~mL} / \mathrm{min}, 250 \mathrm{~nm}\right)$ : purity $90.78 \% ; t_{R} 4.62$ min;

Anal. (\%): Calcd. for $\mathrm{C}_{16} \mathrm{H}_{12} \mathrm{BrNO}_{4} \mathrm{~S}(394.24 \mathrm{~g} / \mathrm{mol})$ : C, 48.74; H, 3.07; N, 3.55; S, 8.13. Found: C, 48.85; H, 3.01; N, 3.49; S, 8.19 .

\section{Synthesis of 2-[4-(4-bromophenylsulfonyl) benzamido] propanoyl chloride 5}

2-[4-(4-Bromophenylsulfonyl) benzamido]propanoic acid $\mathbf{3}(2.27 \mathrm{~g}, 5.5 \mathrm{mmol})$ was refluxed with 25 -fold molar excess of thionyl chloride $(10 \mathrm{~mL})$ on a water bath until emission of sulfur dioxide and hydrogen chloride gas ceased. Unreacted $\mathrm{SOCl}_{2}$ was removed to dryness by distillation under reduced pressure on a water bath. The yellow crystalline crude product was used without further purification; $98 \%$ yield; m.p. $156-158^{\circ} \mathrm{C}$;

FT-IR $\left(\mathrm{KBr}, v \mathrm{~cm}^{-1}\right): 3345 \mathrm{~m}, 3090 \mathrm{~m}, 3067 \mathrm{~m}, 2989 \mathrm{~m}$, $2951 \mathrm{~m}, 2843 \mathrm{w}, 1826 \mathrm{~s}, 1788 \mathrm{~s}, 1651 \mathrm{vs}, 1599 \mathrm{~m}, 1573 \mathrm{vs}$, $1472 \mathrm{~m}, 1524 \mathrm{~s}, 1326 \mathrm{vs}, 1292 \mathrm{vs}, 1160 \mathrm{vs}, 851 \mathrm{~m}, 886 \mathrm{~m}$, 575vs.

General procedures for the synthesis of N-(1-aryl-1oxopropan-2-yl)-4-(4-bromophenylsulfonyl)benzamides 6

Method 1. Anhydrous $\mathrm{AlCl}_{3}(2.0 \mathrm{~g}, 15 \mathrm{mmol})$ was added portionwise under stirring at room temperature to the crude azlactone $4(1.97 \mathrm{~g}, 5 \mathrm{mmol})$ in excess of dry aromatic hydrocarbon $(25 \mathrm{~mL})$. The reaction mixture was stirred for $20 \mathrm{~h}$ and then poured over $100 \mathrm{~mL}$ ice-water with $5 \mathrm{~mL}$ concentrated $\mathrm{HCl}$. The precipitate of crude product was filtered off and washed with cold water and a cool mixture of water-ethanol $(1: 1, v / v)$. The layers of the filtrate were separated and the aqueous phase was extracted twice with $15 \mathrm{~mL} \mathrm{CH} \mathrm{Cl}_{2}$. The combined organic layers were washed with water, dried $\left(\mathrm{Na}_{2} \mathrm{SO}_{4}\right)$ and evaporated under reduced pressure, leaving a second fraction of crude product. Recrystallization from cyclohexane or ethanol supplied the title products as colourless crystals.

Method 2. $2.0 \mathrm{~g}$ ( $15 \mathrm{mmol}$ ) $\mathrm{AlCl}_{3}$ were added portionwise at ambient temperature to the crude 2-[4-(4-bromophenylsulfonyl) benzamido] propanoyl chloride $5(2.15 \mathrm{~g}, 5$ $\mathrm{mmol}$ ) in $25 \mathrm{~mL}$ of dry aromatic hydrocarbon (as solvent and reactant). Stirring was continued until the $\mathrm{HCl}$ was not 
longer produced $(\approx 20 \mathrm{~h})$ and then the reaction mass was poured over $100 \mathrm{~mL}$ mixture of acidulated $(\mathrm{HCl})$ ice-water. After extraction in $\mathrm{CH}_{2} \mathrm{Cl}_{2}$ the organic layer was washed with $5 \% \mathrm{NaHCO}_{3}$ solution, then with water and dried over $\mathrm{Na}_{2} \mathrm{SO}_{4}$. Evaporation of the solvent mixture under reduced pressure and recrystallization of crude products led to colourless solids.

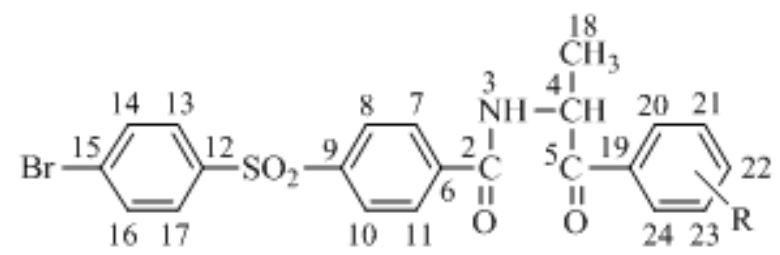

$6 \mathbf{a ~ R}=\mathrm{H} ; \mathbf{6} \mathbf{b ~ R}=4-\mathrm{CH}_{3} ; \mathbf{6} \mathbf{c} \mathrm{R}=2,4-\left(\mathrm{CH}_{3}\right)_{2}$

Fig. 3. The general structure of compounds 6 with atom numbering (for NMR assignments)

4-(4-Bromophenylsulfonyl)-N-(1-oxo-1-phenylpropan-2yl) benzamide $6 a$, obtained by reaction with benzene; $96 \%$ yield (method 1 ), $80 \%$ yield (method 2); m.p. $127-129^{\circ} \mathrm{C}$ (cyclohexane);

UV-Vis ( $\left.\mathrm{CH}_{3} \mathrm{OH}, \lambda \mathrm{nm}\right)(\mathrm{lg} \varepsilon): 203.5$ (4.46), 251.1 (4.35);

FT-IR $\left(\mathrm{KBr}^{3} \vee \mathrm{cm}^{-1}\right)$ : 3347s, 3088m, 3063m, 2983m, 2937m, 2876w, 1693s, 1650vs, 1598s, 1573vs, 1521vs, $1485 \mathrm{~s}, 1450 \mathrm{~s}, 1537 \mathrm{vs}, 1324 \mathrm{vs}, 1292 \mathrm{vs}, 1159 \mathrm{vs}, 854 \mathrm{~m}, 577 \mathrm{~s}$;

${ }^{1} \mathrm{H}-\mathrm{NMR}(\mathrm{DMSO}-\mathrm{d}, \delta \mathrm{ppm}, J \mathrm{~Hz}): 1.38(\mathrm{~d}, 7.0,3 \mathrm{H}, \mathrm{H}-18)$, 5.50 (quint, 7.0, $1 \mathrm{H}, \mathrm{H}-4$ ) , 7.53 (br t, 7.4, 2H, H-21, H-23), 7.64 (brt $7.4,1 \mathrm{H}, \mathrm{H}-22$ ), 7.85 (d, 9.1, 2H, H-14, H-16), 7.91 (d, 9.1, 2H, H-13, H-17), 7.99 (dd, 1.7, 7.4, 2H, H-20, H-24), 8.04 (d, 8.8, 2H, H-7, H-11), 8.08 (d, 8.8, 2H, H-8, H-10), $9.10(\mathrm{~d}, 7.0,1 \mathrm{H}, \mathrm{NH})$;

${ }^{13}$ C-NMR (DMSO-d ${ }_{6}, \delta$ ppm): 16.69 (C-18), 50.61 (C-4), 127.68 (C-8, C-10), 128.28 (C-21, C-23), 128.80 (C-15), 128.85 (C-7, C-11), 128.91 (C-13, C-17), 129.55 (C-20, C24), 133.03 (C-14, C-16), 133.41 (C-22), 134.91 (C-19), 138.63 (C-6), 139.96 (C-12), 142.89 (C-9), 164.73 (C-2), 198.90 (C-5):

RP-HPLC ( $\left.\mathrm{CH}_{2} \mathrm{OH}: \mathrm{H}_{2} \mathrm{O}=60: 40,1 \mathrm{~mL} / \mathrm{min}, 250 \mathrm{~nm}\right)$ : purity $97.46 \%$; $t_{0} 5.80 \mathrm{~min}$;

Anal. (\%): Câlcd. for $\mathrm{C}_{22} \mathrm{H}_{13}$ BrNO S $(472.35 \mathrm{~g} / \mathrm{mol}): \mathrm{C}$, 55.94; H, 3.84; N, 2.97; S, 6.79. Found: C, 55.97; H, 3.80; N, 2.95; S, 6.79 .

4-(4-Bromophenylsulfonyl)-N-(1-oxo-1-p-tolylpropan-2$y$ l) benzamide $\boldsymbol{6} \boldsymbol{b}$, obtained by reaction with toluene; $97 \%$ yield (method 1 ), $86 \%$ yield (method 2); m.p. $140-143^{\circ} \mathrm{C}$ (ethanol);

UV-Vis ( $\left.\mathrm{CH}_{3} \mathrm{OH}, \lambda \mathrm{nm}\right)(\mathrm{lg} \varepsilon): 203.5$ (4.47), $255.5(4.40)$;

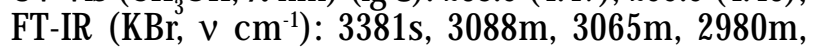
$2937 \mathrm{~m}, 2875 \mathrm{w}, 1686 \mathrm{vs}, 1651 \mathrm{vs}, 1606 \mathrm{~s}, 1573 \mathrm{~s}, 1485 \mathrm{~s}$, $1450 \mathrm{~m}, 1525 \mathrm{vs}, 1323 \mathrm{vs}, 1293 \mathrm{~s}, 1158 \mathrm{vs}, 853 \mathrm{~m}, 576 \mathrm{vs}$;

${ }^{1} \mathrm{H}-\mathrm{NMR}$ (DMSO-d $\left.\mathrm{d}^{\prime} \delta \mathrm{ppm}, J \mathrm{~Hz}\right): 1.39(\mathrm{~d}, 7.1,3 \mathrm{H}, \mathrm{H}-18)$, $2.36\left(\mathrm{~s}, 3 \mathrm{H}, \mathrm{CH}_{3}\right), 5.49$ (quint, 7.1, $\left.1 \mathrm{H}, \mathrm{H}-4\right), 7.33$ (d, 8.2, $2 \mathrm{H}$, $\mathrm{H}-21, \mathrm{H}-23$ ) , 7.85 (d, 8.8, 2H, H-14, H-16), 7.91 (d, 8.8, 2H, $\mathrm{H}-13, \mathrm{H}-17), 7.92$ (d, 8.2, 2H, H-20, H-24), 8.02 (d, 8.5, 2H, H-7, H-11), 8.09 (d, 8.5, 2H, H-8, H-10), 9.10 (d, 7.1, 1H, $\mathrm{NH})$;

${ }^{13} \mathrm{C}-\mathrm{NMR}$ (DMSO-d $\left.\mathrm{d}, \delta \mathrm{ppm}\right): 16.72(\mathrm{C}-18), 21.10\left(\mathrm{CH}_{3}\right)$, 50.34 (C-4), 127.56 ( $\left.C^{\prime}-8, C-10\right), 128.17$ (C-15), 128.30 ( $C^{3}-$ $13, C-17), 128.80$ (C-7, C-11), 129.28 (C-21, C-23), 129.43 (C-20, C-24), 132.19 (C-19), 132.91 (C-14, C-16), 138.56 (C-6), 139.85 (C-12), 142.76 (C-22), 143.74 (C-9), 164.56 (C-2), 198.22 (C-5);

RP-HPLC ( $\left.\mathrm{CH}_{3} \mathrm{OH}: \mathrm{H}_{2} \mathrm{O}=60: 40,1 \mathrm{~mL} / \mathrm{min}, 250 \mathrm{~nm}\right)$ : purity $92.28 \% ; t_{\mathrm{R}} 7.38 \mathrm{~min}$;

Anal. (\%): Câlcd. for $\mathrm{C}_{2} \mathrm{H}_{2}$ BrNO S $(486.38 \mathrm{~g} / \mathrm{mol}):$, 56.80; $H, 4.14 ; \mathrm{N}, 2.88 ; \mathrm{S}, 6.59$. Found: $\mathrm{C}, 56.88 ; \mathrm{H}, 4.20 ; \mathrm{N}$, 2.82; S, 6.62 .
4-(4-Bromophenylsulfonyl)-N-[1-(2,4-dimethylphenyl)-1oxopropan-2-yl]benzamide $\mathbf{6 c}$, obtained by reaction with $m$-xylene; $98 \%$ yield (method 1 ), $87 \%$ yield (method 2 ); m.p. $121-123^{\circ} \mathrm{C}$ (ethanol);

UV-Vis $\left(\mathrm{CH}_{3} \mathrm{OH}, \lambda \mathrm{nm}\right)$ ( $(\mathrm{lg} \varepsilon): 203.5$ (4.46), 255.5 (4.34); FT-IR $\left(\mathrm{KBr}^{3} \vee \mathrm{cm}^{-1}\right)$ : 3396s, 3093m, 3066m, 2983m, $2927 \mathrm{~m}, 2876 \mathrm{~m}, 1695 \mathrm{vs}, 1666 \mathrm{vs}, 1612 \mathrm{~s}, 1573 \mathrm{vs}, 1486 \mathrm{~s}$, 1450s, 1530vs, 1322vs, 1293vs, 1156vs, 853m, 573vs;

${ }^{1} \mathrm{H}-\mathrm{NMR}(\mathrm{DMSO}-\mathrm{d}, \delta \mathrm{ppm}, J \mathrm{~Hz}): 1.32(\mathrm{~d}, 7.1,3 \mathrm{H}, \mathrm{H}-18)$, $2.24\left(\mathrm{~s}, 3 \mathrm{H}, \mathrm{CH}_{3}\right), 2.35\left(\mathrm{~s}, 3 \mathrm{H}, \mathrm{CH}_{3}\right), 5.26$ (quint, $7.1,1 \mathrm{H}, \mathrm{H}-$ 4), $7.10(\mathrm{~m}, 2 \mathrm{H}, \mathrm{H}-21, \mathrm{H}-23), 7.73$ (d, 8.4, $1 \mathrm{H}, \mathrm{H}-24), 7.84$ (d, 8.8, 2H, H-14, H-16), 7.91 (d, 8.8, 2H, H-13, H-17), 8.02 (d, 8.8, 2H, H-7, H-11), 8.08 (d, 8.8, 2H, H-8, H-10), 9.09 (d, 7.1, 1H, NH);

${ }^{13} \mathrm{C}-N M R$ (DMSO-d $\mathrm{d}_{\mathrm{f}^{\prime}} \delta \mathrm{ppm}$ ): 16.01 (C-18), $20.23\left(\mathrm{CH}_{3}\right)$, $20.82\left(\mathrm{CH}_{3}\right), 52.61(\mathrm{C}-4), 126.09(\mathrm{C}-23), 127.56(\mathrm{C}-8, \mathrm{C}-$ 10), 128.17 (C-15), 128.34 (C-24), 128.74 (C-7, C-11), 129.43 (C-13, C-17), 132.18 (C-21), 132.90 (C-14, C-16), 133.41 (C-19), 137.64 (C-20), 138.60 (C-6), 139.84 (C-22), 141.18 (C-12), 142.74 (C-9), 164.69 (C-2), 202.27 (C-5);

+ ESI-MS/MS (m/z, rel. abund. \%): $500\left({ }^{79} \mathrm{Br}\right) / 502\left({ }^{81} \mathrm{Br}\right)$ $[\mathrm{M}+\mathrm{H}]^{+} ; 394 / 396$ (35.6/40.1) [M+H-m-xylene $]^{+} ; 366 / 368$ (100, BP) [M+H-m-xylene-CO]+; 323/325 (47.1/31.8)

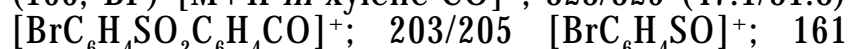
$\left[\mathrm{C}_{8} \mathrm{H}_{6} \mathrm{COCCHCH}_{3}^{4}\right]^{4} ; 155 / 157\left[\mathrm{BrC}_{6} \mathrm{H}_{4}\right]^{+} ; 133\left[\mathrm{C}_{8}^{4} \mathrm{H}_{9} \mathrm{CO}\right]^{+} ; 124$ $\left[\mathrm{C}_{6}^{8} \mathrm{H}^{9} \mathrm{SO}^{+} ; 76\left[\mathrm{C}_{6} \mathrm{H}_{4}\right]^{+}\right.$;

${ }^{6}$-ESI-MS/MS (m/z, rel. abund. \%): $498\left({ }^{79} \mathrm{Br}\right) / 500\left({ }^{81} \mathrm{Br}\right)$ [M-H]; 366/368 [M-H-C $\left.\mathrm{H}_{8} \mathrm{CO}\right] ; 2$ 295/297 (100, BP) [ $\left.\mathrm{BrC}_{\mathrm{H}} \mathrm{SO}_{2} \mathrm{CH}_{4}\right] ; 219 / 221^{8}(65.9 / 38.0)\left[\mathrm{BrCH}^{2} \mathrm{SO}_{2}\right] ; 202$ (89.7/81.8) [ $\left.\mathrm{C}_{8} \mathrm{H}_{0} \mathrm{COCCH}_{3} \mathrm{NCO}\right] ; 174\left[\mathrm{C}_{8} \mathrm{H}_{0} \mathrm{COC}^{4} \mathrm{CCH}_{3}^{2} \mathrm{~N}\right] ;$

RP-HPLC ( $\left.\mathrm{CH}_{3} \mathrm{OH}: \mathrm{H}_{2} \mathrm{O}=60: 40,1 \mathrm{~mL} / \mathrm{min}, 25 \mathrm{~nm}\right)$ : purity $97.55 \% ;$; 6.97 min;

Anal. (\%): Câlcd. for $\mathrm{C}_{22} \mathrm{H}_{22}$ BrNO S $(500.40 \mathrm{~g} / \mathrm{mol})$ : $\mathrm{C}$, 57.60; H, 4.43; N, 2.80; S, 6.41. Found: $C, 57.65 ; H, 4.37 ; \mathrm{N}$, 2.85; S, 6.36 .

General procedures for the synthesis of 5-aryl-2-[4-(4bromophenylsulfonyl)phenyl]-4-methyloxazoles 7

Method 1. The crude N-(1-aryl-1-oxopropan-2-yl)-4-(4bromophenylsulfonyl) benzamides $6(10 \mathrm{mmol})$ were refluxed in $20 \mathrm{~mL}$ phosphorus oxychloride for $4 \mathrm{~h}$. The excess of $\mathrm{POCl}_{3}$ was removed under vacuum. After cooling, the oily residue was treated with a mixture of ice-water and extracted twice with $20 \mathrm{~mL} \mathrm{CH} \mathrm{Cl}_{2}$. The organic layers were combined and washed several times with $5 \% \mathrm{NaHCO}_{3}$ solution, then with water and dried $\left(\mathrm{Na}_{2} \mathrm{SO}_{4}\right)$. After evaporation of the solvent, the crude products were recrystallized from ethanol as colourless needle-shaped crystals.

Method 2. The N-(1-aryl-1-oxopropan-2-yl)-4-(4bromophenylsulfonyl)benzamides $6(10.51 \mathrm{mmol})$ were dissolved in $40 \mathrm{~mL}$ ethyl acetate. Acetic anhydride $(3 \mathrm{~mL}$, $31.75 \mathrm{mmol})$ and $98 \%$ sulfuric acid $(0.17 \mathrm{~mL}, 3.19 \mathrm{mmol})$ in $2.5 \mathrm{~mL}$ ethyl acetate were added. The reaction mass was heated at reflux for $3 \mathrm{~h}$. After cooling to room temperature, a $2.52 \mathrm{~N} \mathrm{NaOH}$ solution $(25 \mathrm{~mL}$ ) was added. The reaction mixture was heated at reflux for another 30 min and then cooled to room temperature. The obtained precipitate was filtered off and washed with cool $1 \mathrm{~N} \mathrm{HCl}$, then with cool $10 \% \mathrm{NaCl}$ solution and finally, with cool water. The layers of filtrate were separated and the organic layer was washed with $1 \mathrm{~N} \mathrm{HCl}$, then with $10 \% \mathrm{NaCl}$ solution, dried over $\mathrm{Na}_{2} \mathrm{SO}_{4}$, and evaporated under vacuum, leaving a second fraction of crude product. The high purity colourless crystals of title compounds were obtained after purification. 


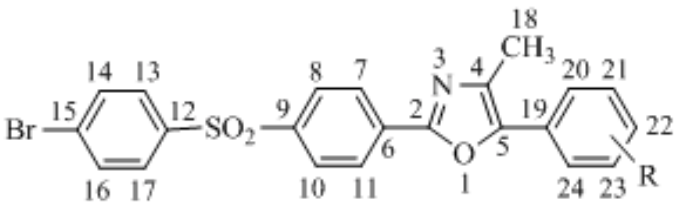

$7 \mathbf{a ~ R}=\mathrm{H} ; 7 \mathbf{b} \mathrm{R}=4-\mathrm{CH}_{3} ; \mathbf{7} \mathbf{c ~ R}=2,4-\left(\mathrm{CH}_{3}\right)_{2} ; \mathbf{7 d ~ R}=2,4,6-\left(\mathrm{CH}_{3}\right)_{3}$

Fig. 4. The general structure of compounds 7 with atom numbering (for NMR assignments)

2-[4-(4-Bromophenylsulfonyl)phenyl]-4-methyl-5phenyloxazole 7a

$92 \%$ yield (method 1 ), $95 \%$ yield (method 2 ); m.p. 177$179{ }^{\circ} \mathrm{C}$ (ethanol);

UV-Vis $\left(\mathrm{CH}_{3} \mathrm{OH}, \lambda \mathrm{nm}\right)(\mathrm{lg} \varepsilon): 203.5$ (4.47), 251.5 (4.26), 337.4 (4.31);

FT-IR $\left(\mathrm{KBr}, v \mathrm{~cm}^{-1}\right): 3087 \mathrm{~m}, 3062 \mathrm{~m}, 2932 \mathrm{~m}, 2863 \mathrm{w}$, $1594 \mathrm{~s}, 1572 \mathrm{~s}, 1544 \mathrm{~m}, 1495 \mathrm{~s}, 1470 \mathrm{~m}, 1444 \mathrm{~m}, 1325 \mathrm{vs}$, 1289s, 1280s, 1155vs, 1093vs, 843s, 573vs;

${ }^{1} \mathrm{H}-\mathrm{NMR}\left(\mathrm{CDCl}{ }_{3}, \delta \mathrm{ppm}, J \mathrm{~Hz}\right): 2.50(\mathrm{~s}, 3 \mathrm{H}, \mathrm{H}-18), 7.37(\mathrm{tt}$, $8.0,1.4,1 \mathrm{H}, \mathrm{H}-22$ ), 7.49 (t, 8.0, 2H, H-21, H-23), 7.67 (d, 8.8, $2 \mathrm{H}, \mathrm{H}-14, \mathrm{H}-16), 7.67$ (dd, 1.4, 8.0, 2H, H-20, H-24), 7.84 (d, 8.8, 2H, H-13, H-17), 8.01 (d, 8.5, 2H, H-7, H-11), 8.19 (d, 8.5, 2H, H-8, H-10);

$\left.{ }^{13} \mathrm{C}-\mathrm{NMR}(\mathrm{CDCl}) \delta \mathrm{ppm}\right): 13.94$ (C-18), 126.03 (C-20, C24), 127.33 (C-8, C-10), 128.71 (C-7, C-11, C-22), 129.21 (C-15), 129.40 (C-21, C-23), 129.72 (C-13, C-17), 132.30 (C-6), 133.20 (C-14, C-16), 134.66 (C-19), 140.87 (C-12), 142.41 (C-9), 147.35 (C-4), 157.77 (C-5), 176.04 (C-2); $\mathrm{RP}-\mathrm{HPLC}\left(\mathrm{CH}_{3} \mathrm{OH}: \mathrm{H}_{2} \mathrm{O}=70: 30,1 \mathrm{~mL} / \mathrm{min}, 335 \mathrm{~nm}\right)$ : purity $98.79 \%$; t $4.67 \mathrm{~min}$;

Anal. (\%): Câlcd. for $\mathrm{C}_{2} \mathrm{H}_{13} \mathrm{BrNO}_{3} \mathrm{~S}(454.34 \mathrm{~g} / \mathrm{mol})$ : C, 58.16; H, 3.55; N, 3.08; S, 7.06. Found: C, 58.11; H, 3.51; N, 3.13; S, 7.02 .

\section{2-[4-(4-Bromophenylsulfonyl)phenyl]-4-methyl-5-p-} tolyloxazole $\mathbf{7 b}$

$94 \%$ yield (method 1), 96\% yield (method 2); m.p. 223-

$224^{\circ} \mathrm{C}$ (ethanol):

UV-Vis $\left(\mathrm{CH}_{3} \mathrm{OH}, \lambda \mathrm{nm}\right)(\mathrm{lg} \varepsilon): 203.5$ (4.47), 249.3 (4.23), 341.9 (4.24);

FT-IR $\left(\mathrm{KBr}, v \mathrm{~cm}^{-1}\right): 3086 \mathrm{~m}, 3066 \mathrm{~m}, 2918 \mathrm{~m}, 2861 \mathrm{~m}$, $1595 \mathrm{vs}, 1574 \mathrm{vs}, 1540 \mathrm{~m}, 1509 \mathrm{vs}, 1473 \mathrm{~s}, 1446 \mathrm{~m}, 1325 \mathrm{vs}$, $1293 \mathrm{vs}, 1278 \mathrm{~s}, 1155 \mathrm{vs}, 1089 \mathrm{vs}, 846 \mathrm{~s}, 566 \mathrm{vs}$;

${ }^{1} \mathrm{H}-\mathrm{NMR}\left(\mathrm{CDCl}_{3}, \delta \mathrm{ppm}, J \mathrm{~Hz}\right): 2.41\left(\mathrm{~s}, 3 \mathrm{H}, \mathrm{CH}_{3}\right), 2.48(\mathrm{~s}$, $3 \mathrm{H}, \mathrm{H}-18), 7.28$ (d, 8.8, 2H, H-21, H-23), 7.57 (d, 8.8, 2H, H20, H-24), 7.67 (d, 8.8, 2H, H-14, H-16), 7.84 (d, 8.8, 2H, H$13, \mathrm{H}-17), 8.01$ (d, 8.8, 2H, H-7, H-11), 8.18 (d, 8.8, 2H, H-8, $\mathrm{H}-10)$;

${ }^{13} \mathrm{C}-\mathrm{NMR}\left(\mathrm{CDCl}{ }_{3}, \delta \mathrm{ppm}\right): 13.53(\mathrm{C}-18), 21.46\left(\mathrm{CH}_{3}\right)$, 125.67 (C-20, C-24), 126.92 (C-8, C-10), 128.36 (C-7, C11), 128.85 (C-15), 129.37 (C-13, C-17), 129.75 (C-21, C23), 132.46 (C-6), 132.86 (C-14, C-16), 133.67 (C-19), 138.46 (C-22), 140.57 (C-12), 141.93 (C-9), 147.25 (C-4), 157.17 (C-5), 175.70 (C-2);

RP-HPLC $\left(\mathrm{CH}_{3} \mathrm{OH}: \mathrm{H}_{2} \mathrm{O}=70: 30,1 \mathrm{~mL} / \mathrm{min}, 335 \mathrm{~nm}\right)$ : purity $97.66 \%$; $5.68 \mathrm{~min}$;

Anal. (\%): Câlcd. for $\mathrm{C}_{2} \mathrm{H}_{18} \mathrm{BrNO}_{3} \mathrm{~S}(468.36 \mathrm{~g} / \mathrm{mol})$ : $\mathrm{C}$, 58.98; H, 3.87; N, 2.99; S, 6.85. Found: C, 58.93; H, 3.82; N, 3.08; S, 6.81 .

\section{2-[4-(4-Bromophenylsulfonyl) phenyl]-5-(2,4-} dimethylphenyl)-4-methyloxazole $7 c$

$93 \%$ yield (method 1 ), $97 \%$ yield (method 2); m.p. 169171 으 (ethanol);

UV-Vis $\left(\mathrm{CH}_{3} \mathrm{OH}, \lambda \mathrm{nm}\right)(\mathrm{lg} \varepsilon): 203.5$ (4.47), 249.3 (4.11), 326.9 (4.09);
FT-IR $\left(\mathrm{KBr}, v \mathrm{~cm}^{-1}\right): 3092 \mathrm{~m}, 3065 \mathrm{w}, 2923 \mathrm{~m}, 2861 \mathrm{w}$, $1601 s, 1572 \mathrm{~s}, 1494 \mathrm{~s}, 1469 \mathrm{~m}, 1448 \mathrm{~m}, 1328 \mathrm{vs}, 1291 \mathrm{~s}$, 1279s, 1159vs, 1096vs, 843s, 571vs;

${ }^{1} \mathrm{H}-\mathrm{NMR}\left(\mathrm{CDCl}_{3}, \delta \mathrm{ppm}\right.$, J Hz): $2.26\left(\mathrm{~s}, 3 \mathrm{H}, \mathrm{CH}_{3}\right), 2.33(\mathrm{~s}$, $\left.3 \mathrm{H}, \mathrm{CH}_{3}\right), 2.38(\mathrm{~s}, 3 \mathrm{H}, \mathrm{H}-18), 7.09$ (br d, 8.0, $\left.1 \mathrm{H}, \mathrm{H}-23\right), 7.14$ (br s, $1 \mathrm{H}, \mathrm{H}-21), 7.25$ (d, 8.0, 1H, H-24), 7.65 (d, 8.5, 2H, H14, H-16), 7.82 (d, 8.5, 2H, H-13, H-17), 7.99 (d, 8.8, 2H, H7, H-11), 8.16 (d, 8.8, 2H, H-8, H-10);

${ }^{13} \mathrm{C}$-NMR (CDCl $\left.{ }_{3}, \delta \mathrm{ppm}\right): 12.53(\mathrm{C}-18), 20.40\left(\mathrm{CH}_{3}\right), 21.38$ $\left(\mathrm{CH}_{3}\right), 126.78$ (C-23), 126.90 (C-8, C-10), 128.41 (C-7, C11), 128.82 (C-15), 129.41 (C-13, C-17), 129.97 (C-24), 131.86 (C-21), 132.36 (C-6), 132.87 (C-14, C-16), 135.07 (C-19), 137.50 (C-20), 139.68 (C-22), 140.77 (C-12), 142.12 (C-9), 147.89 (C-4), 158.07 (C-5), 175.73 (C-2);

+ ESI-MS/MS (m/z, rel. abund. \%): $482\left({ }^{79} \mathrm{Br}\right) / 484\left({ }^{81} \mathrm{Br}\right)$ $[\mathrm{M}+\mathrm{H}]^{+} ; 466 / 468\left[\mathrm{M}+\mathrm{H}-\mathrm{CH}_{3}\right]^{+} ; 323 / 325\left[\mathrm{BrC}^{\mathrm{H}} \mathrm{SO}\right.$ $\left.\mathrm{C}_{6} \mathrm{H}_{4} \mathrm{CO}\right]^{+} ; 279\left[\mathrm{M}+\mathrm{H}-\mathrm{BrCl}_{6} \mathrm{HSO}^{+} ; 263(100, \mathrm{BP})\left[\mathrm{M}^{4}+\mathrm{H}^{2}\right.\right.$ $\left.\mathrm{BrC}_{6}^{6} \mathrm{H}_{4} \mathrm{SO}_{2}\right]^{+} ; 248\left[\mathrm{M}+\mathrm{H}-\mathrm{CH}_{3}-\mathrm{BrC}_{6} \mathrm{H}_{5} \mathrm{SO}_{2}\right]^{+} ; 158[\mathrm{M}+\mathrm{H}-$ $\left.\mathrm{BrC}^{6} \mathrm{H}^{4} \mathrm{SO}_{2}-m-x y l y l\right]^{+}$;

$\mathrm{RP}^{6} \mathrm{HPLC}\left(\mathrm{CH}_{3} \mathrm{OH}: \mathrm{H}_{2} \mathrm{O}=70: 30,1 \mathrm{~mL} / \mathrm{min}, 335 \mathrm{~nm}\right)$ : purity $96.80 \% ; t_{R} 5.68$ min;

Anal. (\%): Câlcd. for $\mathrm{C}_{2} \mathrm{H}_{2}$ BrNO $_{3} \mathrm{~S}(482.39 \mathrm{~g} / \mathrm{mol}): \mathrm{C}$, 59.76; H, 4.18; N, 2.90; S, 6.65. Found: C, 59.82; H, 4.11; N, $2.84 ; \mathrm{S}, 6.68$.

\section{2-[4-(4-Bromophenylsulfonyl)phenyl]-5-mesityl-4- methyloxazole $7 \boldsymbol{d}$}

$89 \%$ yield (method 1 ), $92 \%$ yield (method 2); m.p. $155-$ $157^{\circ} \mathrm{C}$ (ethanol);

UV-Vis $\left(\mathrm{CH}_{3} \mathrm{OH}, \lambda \mathrm{nm}\right)(\mathrm{lg} \varepsilon): 204.4$ (4.47), 249.3 (4.21), 324.2 (4.13);

FT-IR $\left(\mathrm{KBr}, v \mathrm{~cm}^{-1}\right): 3089 \mathrm{w}, 3066 \mathrm{w}, 2921 \mathrm{~m}, 2863 \mathrm{w}$, $1600 \mathrm{~s}, 1573 \mathrm{~s}, 1499 \mathrm{~m}, 1472 \mathrm{~m}, 1455 \mathrm{~m}, 1325 \mathrm{~s}, 1290 \mathrm{~s}$, $1159 \mathrm{vs}, 1101 \mathrm{~s}, 845 \mathrm{~m}, 575 \mathrm{~s}$;

${ }^{1} \mathrm{H}-\mathrm{NMR}\left(\mathrm{CDCl}_{3}, \delta \mathrm{ppm}, J \mathrm{~Hz}\right): 2.27\left(\mathrm{~s}, 3 \mathrm{H}, \mathrm{CH}_{3}\right), 2.29(\mathrm{~s}$, $\left.6 \mathrm{H}, \mathrm{CH}_{3}\right), 2.38(\mathrm{~s}, 3 \mathrm{H}, \mathrm{H}-18), 7.10(\mathrm{~s}, 1 \mathrm{H}, \mathrm{H}-23), 7.13(\mathrm{~s}, 1 \mathrm{H}$, $\mathrm{H}-21), 7.66(\mathrm{~d}, 8.5,2 \mathrm{H}, \mathrm{H}-14, \mathrm{H}-16), 7.83(\mathrm{~d}, 8.5,2 \mathrm{H}, \mathrm{H}-13$, $\mathrm{H}-17), 7.99$ (d, 8.8, 2H, H-7, H-11), 8.17 (d, 8.8, 2H, H-8, H10);

${ }^{13} \mathrm{C}-\mathrm{NMR}(\mathrm{CDCl}, \delta \mathrm{ppm}): 12.57(\mathrm{C}-18), 19.31\left(\mathrm{CH}_{3}\right), 19.68$ $\left(\mathrm{CH}_{3}\right), 19.88\left(\mathrm{CH}_{3}\right), 126.83(\mathrm{C}-8, \mathrm{C}-10), 128.37(\mathrm{C}-7, \mathrm{C}-11)$, 128.82 (C-15), 129.36 (C-13, C-17), 130.96 (C-23), 132.28 (C-6), 132.38 (C-21), 132.84 (C-14, C-16), 134.19 (C-19), 138.30 (C-20, C-24), 139.13 (C-22), 140.58 (C-12), 141.91 (C-9), 147.90 (C-4), 157.95 (C-5), 175.70 (C-2);

RP-HPLC $\left(\mathrm{CH}_{3} \mathrm{OH}: \mathrm{H}_{2} \mathrm{O}=70: 30,1 \mathrm{~mL} / \mathrm{min}, 335 \mathrm{~nm}\right)$ : purity $90.58 \%, t \mathrm{~b} .02 \mathrm{~min}$;

Anal. (\%): Calcd. for $\mathrm{C}_{25} \mathrm{H}_{22} \mathrm{BrNO}_{3} \mathrm{~S}(496.42 \mathrm{~g} / \mathrm{mol}): \mathrm{C}$, $60.49 ; \mathrm{H}, 4.47 ; \mathrm{N}, 2.82 ; \mathrm{S}, 6.46$. Found: $\mathrm{C}, 60.54 ; \mathrm{H}, 4.47 ; \mathrm{N}$, 2.85; S, 6.42 .

\section{Cytoxicity evaluation}

The Daphnia magna bioassay was performed under constant temperature and light conditions (at $25 \pm 1{ }^{\circ} \mathrm{C}$, in the dark) using a Sanyo MLR-351 H, USA climatic chamber.

The determinations were made in duplicate against áalanine (positive control) and 1\% DMSO (negative control). The experiment was carried out according to the protocol previously described [27-29]. From each compound, six concentrations ranging from 5 to $200 \mu \mathrm{g} / \mathrm{mL}$ were tested. The lethality curves were plotted using the logarithm of concentrations and against lethality percentage, L (\%), recorded at 24,48 and $72 \mathrm{~h}$. The prediction was performed with the GUSAR software application.

\section{Results and discussions}

\section{Chemistry}

In the light of the above importance of oxazol-5(4H)ones and oxazoles, is seems of interest to synthesize new 
heterocyclic compounds from these classes and their acyclic intermediates using the reaction sequences from scheme 1. The key precursor, 4-(4-bromophenylsulfonyl) benzoic acid $\mathbf{1}$, and corresponding acyl chloride $\mathbf{2}$ were already described in literature [30,31]. Compound 1 was synthesized by Friedel-Crafts reaction between bromobenzene and 4-methylbenzene-1-sulfonyl chloride ( $p$-tosyl chloride) in the presence of anhydrous $\mathrm{AlCl}_{3}$ at reflux, followed by oxidation of 4-(4-bromophenylsulfonyl)1-methylbenzene with chromium trioxide in glacial acetic acid at reflux [30]. The acid 1 was then converted by reaction with $\mathrm{SOCl}_{\text {, into }}$-(4-bromophenylsulfonyl) benzoyl chloride 2 [20,21] which was used without further purification for $\mathrm{N}$-acylating á-alanine according to Steiger's procedure in order to obtain 2-[4-(4-bromophenylsulfonyl) benzamido]propanoic acid $\mathbf{3}$. This compound was then cyclodehydrated to the corresponding azlactone 4 by two methods using either ethyl chloroformate in the presence of $\mathrm{N}$-methylmorpholine in methylene chloride at room temperature or acetic anhydride at reflux. Cyclization in basic medium may be considered to take place according to the similar mechanism to that we previously described for other 2,4-disubstituted-5(4H)-oxazolone [20].

The $N$-acylated amino acid $\mathbf{3}$ was also converted through a nucleophilic substitution reaction with excess of thionyl dichloride at reflux into the corresponding acyl chloride 5 .

The $\mathrm{AlCl}_{3}$-catalyzed acylaminoacylation of the aromatic hydrocarbons (in excess both as reactant and solvent) with $5(4 \mathrm{H})$-oxazolone 4 was carried outatambient temperature and led to the $\alpha$-acylamino ketones 6 , with a high regioselectivity and at excellent yields - which increase in the order: benzene, toluene, $m$-xylene, in agreement with the increasing nucleophilicity of these substrates and the stability of corresponding Wheland intermediate in electrophilic aromatic substitution (EAS). The proposed ring opening reaction mechanism is formerly indicated by us in the literature [21]. Compounds 6 have also obtained by Friedel-Crafts acylation of aromatic hydrocarbons with 2[4-(4-bromophenylsulfonyl) benzamido]propanoyl chloride 5 , butthe reaction yields were lower. These results indicate that $5(4 H)$-oxazolones are better $N$-acylating reagents than $N$-acyl- $\alpha$-amino acid chlorides.

In the Robinson-Gabriel synthesis conditions, by using phosphoryl trichloride or concentrated sulfuric acid in the presence of acetic anhydride in ethyl acetate, the above $\mathrm{N}$-(1-aryl-1-oxopropan-2-yl)-4-(4-bromophenylsulfonyl) benzamides 6a-c were cyclodehydrated affording 2,5diaryl-4-methyloxazoles 7a-c in very good yields.

Generally, intermediate compounds from $\alpha$-acilamino ketones class (6a-c) were isolated as pure colourless crystals and characterized physico-chemically, with the exception of 4-(4-bromophenylsulfonyl)- $\mathrm{N}$-(1-mesityl-1oxopropan-2-yl) benzamide $\mathbf{6 d}$ (obtained by reaction with mesitylene), which could not be isolated in pure form, but which has been used in crude state in the synthesis of the corresponding oxazole 7d.

The proposed mechanism for synthesis of 2,5-diaryl-4methyloxazoles 7 from 2-aza-1,4-diaryl-3-methyl-1,4butanediones 6 in the presence of excess phosphorus oxychloride occurs via the enolized forms I and then through the ester-dichloride intermediates of phosphoric acid II. The leaving group dichlorophosphate, $-\mathrm{OPOCl}_{2}$, is replaced by chloride anion by bimolecular nucleophilic substitution mechanism in order to form chlorinated compounds III. The unstable chloride anions IV are then obtained, which lead to the formation of the compounds with oxazole ring 7 by intramolecular nucleophilic addition accompanied by elimination of chloride anion (scheme 2.a). In acid medium, the reaction mechanism for obtaining 1,3-oxazoles 7 involves the protonation of compounds 6 with the formation of two electrophilic structures in resonance: oxonium ions $\mathbf{V}$ and corresponding carbocations VI. The carbocations VI were than deprotonated simultaneously with the cyclization by nucleophilic attack at C-4, leading to the corresponding unstable hemiketals (2,5-diaryl-5-hydroxy-4-methyl-4,5dihydrooxazoles) VII. An intramolecular dehydration reaction of these intermediates affords heterocyclic compounds from 1,3-oxazoles class 7 (scheme 2.b). This mechanism is in accordance to literature data [32], based on ${ }^{18} \mathrm{O}$-labelling, which indicated that the amidic oxygen from acyclic intermediates is maintained in the oxazole ring and the ketonic oxygen is removed as water.

The chemical structures of the new compounds are confirmed due to their spectral (UV-Vis, IR, ${ }^{1} \mathrm{H}-\mathrm{NMR},{ }^{13} \mathrm{C}$ NMR, MS) and elemental analysis.

Generally, the electronic absorption spectra of the new compounds presented a sharp band at $\lambda 202.6-204.4 \mathrm{~nm}$ ( $E$ band) and an absorption at $\lambda$ 249.3-255.5 nm ( $B$ band). In addition, the compounds $\mathbf{3}$ and $\mathbf{4}$ show a third absorption maximum of weak intensity at $\lambda 226.4 \mathrm{~nm}, 228.2 \mathrm{~nm}$, respectively ( $\mathrm{K}$ band). The presence of an additional intense absorption maximum at higher longest-wavelengths, $\lambda$ $324.2-341.9 \mathrm{~nm}$, is observed in the UV spectra of the new oxazoles 7 compared with those of acyclic precursors 6 . This bathochromic shift is due to extending of conjugation by formation of oxazole chromophore.

Presence of the characteristic absorption bands in IR spectra of the synthesized products provides useful information for determining the structure of newly compounds 3-7. Thus, 2-[4-(4-bromophenylsulfonyl) benzamido]propanoic acid 3 and $\mathrm{N}$-(1-aryl-1-oxopropan2-yl)-4-(4-bromophenylsulfonyl) benzamides 6 exhibited the following characteristic absorption bands at wavenumbers: $3347-3396 \mathrm{~cm}^{-1}$ for $\mathrm{N}-\mathrm{H}$ stretching, $\mathrm{v}(\mathrm{N}$ $\mathrm{H})$, at $1686-1708 \mathrm{~cm}^{-1}$ due to carbonyl absorption, $\mathrm{v}(\mathrm{O}=\mathrm{C}$ C), and at 1644-1666 $\mathrm{cm}^{-1}$ due to amidic carbonyl group stretching vibration, $v(\mathrm{O}=\mathrm{C}-\mathrm{N})$ (amide I band). Characteristic of these compounds is also the amide II band, assigned to deformation vibration of $\mathrm{N}-\mathrm{H}$ group, $\delta(\mathrm{N}$ $\mathrm{H})$, present in the region $1523-1537 \mathrm{~cm}^{-1}$. Amide III band due to the stretching vibration of the $\mathrm{C}-\mathrm{N}$ bond, $\mathrm{v}(\mathrm{C}-\mathrm{N})$, and only in compound $\mathbf{3}$, the absorption band attributed to the stretching vibration of the $\mathrm{C}-\mathrm{O}$ bond, $\mathrm{v}(\mathrm{C}-\mathrm{O})$, overlap the absorption bands due to antisymmetric stretching vibration of the sulfonyl group, $\mathrm{v}_{(}\left(\mathrm{SO}_{2}\right)$. In addition, the $\mathrm{O}-\mathrm{H}$ stretching absorption, $v(\mathrm{O}-\mathrm{f} f)$, for hydrogen-bonded dimers of compound $\mathbf{3}$ is strong and very broad, extending from $2500 \mathrm{~cm}^{-1}$ to $3000 \mathrm{~cm}^{-1}$. This absorption overlaps the medium sharper $\mathrm{C}-\mathrm{H}$ stretching peaks, which are extending beyond the $\mathrm{O}-\mathrm{H}$ envelope.

Evidence for the obtaining of acyl chloride 5 are presence in IR spectrum of two strong absorption bands due to $v(O=C-N)$ at $1826 \mathrm{~cm}^{-1}$ (fundamental vibration ) and 1788 $\mathrm{cm}^{-1}$ (Fermi resonance band), and a medium band due to $\mathrm{v}(\mathrm{C}-\mathrm{Cl})$ at $886 \mathrm{~cm}^{-1}$.

The IR spectra of heterocyclic compounds $\mathbf{4}$ and $\mathbf{7}$ were clearly distinguished from those of corresponding acyclic intermediates $\mathbf{3}$ and $\mathbf{6}$, respectively by having different characteristic wavenumbers, in agreement with the literature data $[20,21]$. Thus, in IR spectrum of azlactone 4 , the absorption band due to the valence vibration of carbonyl group was shifted at $1820 \mathrm{~cm}^{-1}$, while the $v(\mathrm{~N}$ $\mathrm{H}), v(\mathrm{O}-\mathrm{H}), v(\mathrm{O}=\mathrm{C}-\mathrm{N})$, and $\delta(\mathrm{N}-\mathrm{H})$ absorption bands from acyclic precursor $\mathbf{3}$ were not observed. Also, the IR spectra of oxazoles 7 revealed the absence of signals in the N-H 


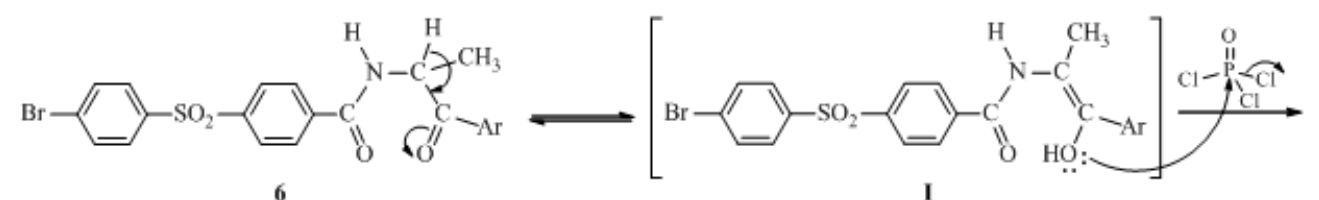

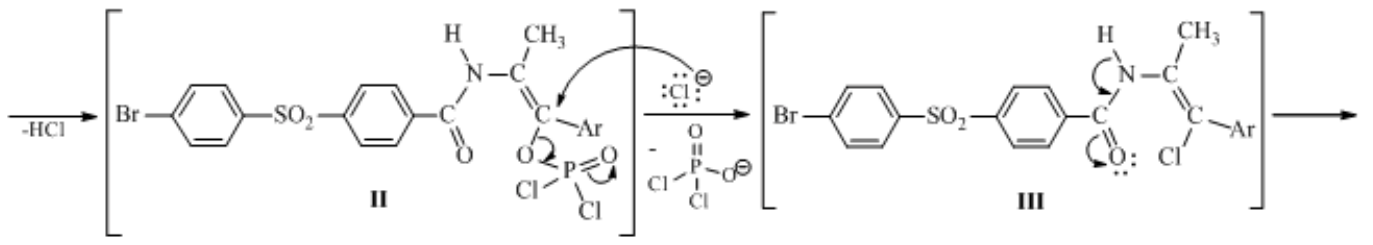

Scheme 2. Proposed mechanisms for obtaining<smiles>[3H][Se]c1ccc([Se]Oc2ccc(-c3nc(C)c([Hg])o3)cc2)cc1</smiles>
1,3-oxazoles in: (a) presence of $\mathrm{POCl}_{3}$ and (b) acid medium.

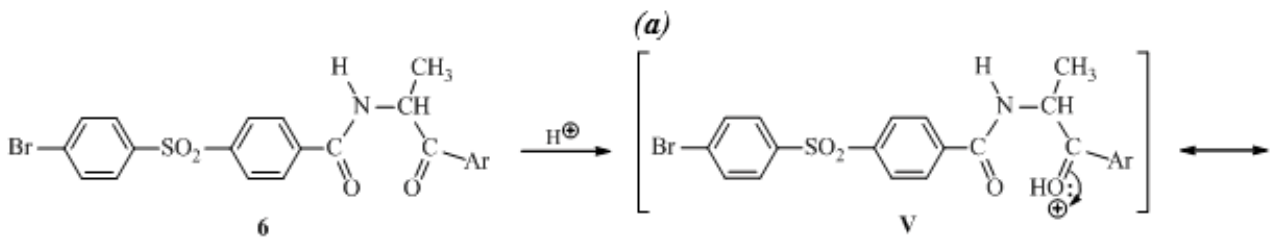

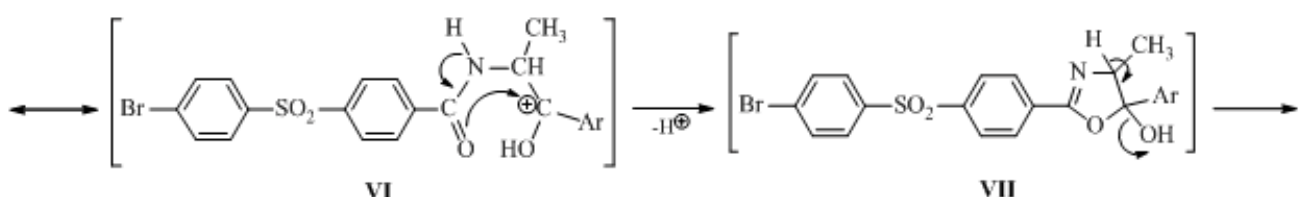<smiles>Cc1nc(-c2ccc(O[SH](=O)(OC3CCCCC3)c3ccc(Br)cc3)cc2)oc1[Al]</smiles>

(b)

and $\mathrm{C}=0$ regions. The peaks at $1650 \mathrm{~cm}^{-1}$ (from 4 ), and in the range 1594-1601 $\mathrm{cm}^{-1}$ (from 7) were assigned to the $\mathrm{C}=\mathrm{N}$ stretching vibration of these new heterocycles.

The formation of compounds $3, \mathbf{4}, \mathbf{6}$ and $\mathbf{7}$ was further confirmed by the ${ }^{1} \mathrm{H}-\mathrm{NMR}$ spectra. Assignments of the signals are based on the chemical shift and intensity pattern. Futhermore, the $2 \mathrm{D}^{1} \mathrm{H}-{ }^{1} \mathrm{H}$ COSY experiments allow unambiguous assignments.

The ${ }^{1} \mathrm{H}-N M R$ spectra of the compounds 3 and $\mathbf{6}$ exhibited a doublet attributed to secondary amide proton at a chemical shift between 8.95-9.10 ppm.

The ${ }^{1} \mathrm{H}-\mathrm{NMR}$ spectra of compounds 4 and 7 contain two sub-spectra characteristic of the $5(4 \mathrm{H})$-oxazolone and oxazole ring, respectively and of the diarylsulfone moiety. The signal attributed to the one proton of the $\mathrm{NH}$ group from acyclic precursors 3 and $\mathbf{6}$ is absent in the ${ }^{1} \mathrm{H}$-NMR spectra of corresponding heterocycles $\mathbf{4}$ and $\mathbf{7}$, respectively and this proves that these new compounds have been obtained.

In the ${ }^{1} \mathrm{H}-\mathrm{NMR}$ spectra of the compounds 3 and $\mathbf{6}$, the methine proton from C-4 appears as a quintet at $4.41 \mathrm{ppm}$ (3) and 5.26-5.50 ppm (6), while for azlactone $\mathbf{4}$ was observed at $4.49 \mathrm{ppm}$ as a quartet and in the case of oxazoles 7 it is absent.

Evidence for the formation of the oxazoles 7 was provided by their ${ }^{1} \mathrm{H}-\mathrm{NMR}$ spectra, which revealed a downfield shift in the signal attributed to the three protons $(\mathrm{H}-18)$ of the methyl group in 4-position from $\delta 1.32-1.39$ ppm in $\alpha$-acylamino ketones 6 as a doublet (because of vicinal couplings with $\mathrm{H}-4$ ) to $2.38-2.50 \mathrm{ppm}$ in oxazoles 7 as a singlet, due to the stronger deshielding effect of oxazole ring compared to that of the $\mathrm{C}=\mathrm{O}$ and $\mathrm{CONH}$ groups from acyclic intermediates $\mathbf{6}$. Also, the methyl doublet in azlactone $\mathbf{4}$ showed a discernible downfield shift of 0.21 ppm relative to the acyclic precursor $\mathbf{3}$, due to the stronger deshielding effect of oxazolone ring compared to that of the $\mathrm{COOH}$ and $\mathrm{CONH}$ groups from compound 3.

The signals in ${ }^{13} \mathrm{C}-\mathrm{NMR}$ spectra are also in good agreement with the proposed structures for the newly synthesized compounds. The assignment of the signals in

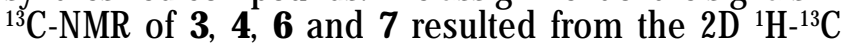
HETCOR experiments.

The chemical shift of the $\mathrm{C}$-4 atom from $\mathrm{N}$-acyl-a-amino acid $\mathbf{3}$ at $48.36 \mathrm{ppm}$ is downfield after intramolecular cyclodehydration to $5(4 H)$-oxazolone 4 with $13.03 \mathrm{ppm}$. Also, in the oxazoles 7 the C-4 signal was more deshielded with $\approx 96 \mathrm{ppm}$ ( $\delta$ 147.25-147.90 ppm) by comparison of the signal of the same atom from $6(\delta 50.34-52.61 \mathrm{ppm})$ and this confirmed that cyclization of the $\alpha$-acylamino ketones 6 took place. It can be noticed the apparition of the downfield signal attributed to the C-2 at $\delta 175.70-176.04$ ppm from the oxazole nucleus, while the carbon atom signal attributed to the amidic carbonyl group from intermediates 6 (in the range 164.56-164.73 ppm) is absent in these compounds. In the ${ }^{13} \mathrm{C}$-NMR spectra of oxazoles 7 , the $\mathrm{C}-5$ atom resonated at $\delta 157.17-158.07 \mathrm{ppm}$, whereas the carbonyl carbon of the compounds 6 resonated at $\delta$ 198.22-202.27 ppm revealing an upfield shift for this carbon in the oxazole structure, which is a further indication that the oxazole formation had taken place. 


\begin{tabular}{|c|c|c|c|}
\hline Compound & $\begin{array}{l}\text { Predicted LC50 } 04 \mathrm{~h} \\
(\mu \mathrm{g} / \mathrm{mL})\end{array}$ & $\begin{array}{l}\text { Determined LC50 } 0 \text { k } \\
(\mu \mathrm{g} / \mathrm{mL})\end{array}$ & $\begin{array}{l}95 \% \text { CI for determined LC5 } 0_{72} \mathrm{~h} \\
(\mu \mathrm{g} / \mathrm{mL})\end{array}$ \\
\hline 3 & 2.2 & 684.9 & $147.4-3182$ \\
\hline 4 & 0.53 & 234.1 & $211.7-258.9$ \\
\hline $6 a$ & 0.39 & 5.38 & $\mathrm{NC}$ \\
\hline $6 \mathrm{~b}$ & 0.3 & 0.71 & $\mathrm{NC}$ \\
\hline $6 c$ & 0.13 & 464.1 & $\mathrm{NC}$ \\
\hline $7 \mathbf{a}$ & 0.11 & 232.0 & $\mathrm{NC}$ \\
\hline $7 \mathrm{~b}$ & 0.08 & $\mathrm{NC}$ & $\mathrm{NC}$ \\
\hline $7 \mathrm{c}$ & 0.06 & $\mathrm{NC}$ & $\mathrm{NC}$ \\
\hline $7 d$ & 0.07 & 127.4 & $102.3-145.3$ \\
\hline$\alpha$-alanine & 1861.3 & $\mathrm{NC}$ & $\mathrm{NC}$ \\
\hline
\end{tabular}

Table 1

RESULTSOF DAPHNIA MAGNA BIOASSAY

LC50 - 50\% lethal concentration; $95 \% \mathrm{CI}$ - 95\% confidence interval; NC - not calculated due to the obtained results

Furthermore, an additional support for the assigned structures of new compounds 3, 4, 6c and 7c was obtained by recording their mass spectra by LC-ESI-MS/MS analysis. Due to the lower polarity, higher stability and volatility compared to the other newly compounds, only $5(4 H)$ oxazolone 4 could be analyzed by GC-EI-MS. The protonated and/or deprotonated molecular ions (LC-ESIMS/MS) or molecular ions (GC-El-MS) (corresponding to the bromine isotopes, ${ }^{79} \mathrm{Br} /{ }^{31} \mathrm{Br}$ ), and main fragments for these new compounds are reported in experimental protocols. The fragmentation pattern is consistent with the structure.

Other characteristic spectral data of new compounds 3-7 are given in the Experimental part.

\section{Cytotoxicity evaluation}

The results of Daphnia magna bioassay are presented in table 1. LC50 could notbe calculated for any of the tested compounds at 24 and $48 \mathrm{~h}$ due to an L\% below $10 \%$. At 72 $\mathrm{h}$, the highest toxicity was induced by compound $\mathbf{6 b}$, followed by $\mathbf{6 a}, \mathbf{7 d}$ and $\mathbf{6 c}$. Compound $\mathbf{4}$ showed toxicity comparable with $\mathbf{7 a}$, whereas compound $\mathbf{3}$ induced an approximately 3 -fold lower toxicity than compound $\mathbf{4}$. As expected, no lethality was recorded for $\alpha$-alanine during the experiment. Compunds belonging to 6 -series presented a higher toxicity as opposed to the 7-series compounds. The predicted values of LC50 for all newly synthetized compounds showed a high toxicity. However, the prediction was confirmed only for compound $\mathbf{6 b}$ and in a lesser extent for compound 6 a.

\section{Conclusions}

Ten newly compounds from $\mathrm{N}$-acyl- $\alpha$-amino acid, $\mathrm{N}$ acyl- $\alpha$-amino acid chloride, 1,3-oxazol-5(4H)-one, $\alpha$ acylamino ketone and 1,3-oxazole class were synthesized and characterized. The new azlactone 4 has been obtained by the reaction of acyl chloride 2 with $\alpha$-alanine, followed by cyclodehydration of the new $N$-acyl- $\alpha$-alanine 3 . The new $\alpha$-acylamino ketones 6 have been obtained by treatment of $5(4 H)$-oxazolone 4 or new $N$-acyl- $\alpha$-alanyl chloride 5 with aromatic hydrocarbons under Friedel-Crafts reaction conditions. Finally, by refluxing these intermediates 6 with phosphorus oxychloride or sulfuric acid in the presence of acetic anhydride, the intramolecular ring closure occurred with formation of the new oxazoles 7. The structure of compounds was confirmed by elemental analysis and different spectral methods.

The new ly synthetized compounds 3, 4, 6a-c, 7a-d have been investigated for their biological activity on Daphnia magna. Compounds $\mathbf{6 a}$ and $\mathbf{6} \mathbf{b}$ showed the highest toxicity, comparable with the predictions performed using GUSAR software. However, further studies are needed in order to investigate the mechanism of action and the therapeutic potential of the compounds.
1. ZHANG, H.-Z., ZHAO, Z.-L., ZHOU, C.-H., Eur. J. Med. Chem., 144, 2018, p. 444.

2. (a) DE GAETANO, M., BUTLER, E., GAHAN, K., ZANETTI, A., MARAI, M., CHEN, J., CACACE, A., HAMS, E., MAINGOT, C., MCLOUGHLIN, A., BRENNAN, E., LEROY, X., LOSCHER, C. E., FALLON, P., PERRETTI, M., GODSON, C., GUIRY, P. J ., Eur. J. Med. Chem., 162, 2019, p. 80. (b) SINGH, N., BHATI, S. K., KUMAR, A., Eur. J. Med. Chem., 43, no. 11, 2008, p. 2597.

3. LIN, S.-Y., KUO, Y.-H., TIEN, Y.-W., KE, Y.-Y., CHANG, W.-T., CHANG, H.-F., OU, L.-C., LAW, P.-Y., XI, J.-H., TAO, P.-L., LOH, H. H., CHAO, Y.S., SHIH, C., CHEN, C.-T., YEH, S.-H., UENG, S.-H., Eur. J. Med. Chem., 167, 2019, p. 312.

4. (a) ANSARI, A., ALI, A., ASIF, M., RAUF, M. A., OWAIS, M., SHAMSUZZAMAN, Steroids, 134, 2018, p. 22. (b) FERNÁNDEZ, L. R., SVETAZ, L., BUTASSI, E., ZACCHINO, S. A., PALERMO, J. A., SÁNCHEZ, M., Steroids, 108, 2016, p. 68.

5. (a) RAVAL, P., JAIN, M., GOSWAMI, A., BASU, S., GITE, A., GODHA, A., PINGALI, H., RAVAL, S., GIRI, S., SUTHAR, D., SHAH, M., PATEL, P., Bioorg. Med. Chem. Lett., 21, no. 10, 2011, p. 3103. (b) PINGALI, $H_{.}$, JAIN, M., SHAH, S., MAKADIA, P., ZAWARE, P., GOEL, A., PATEL, M., GIRI, S., PATEL, H., PATEL, P., Bioorg. Med. Chem., 16, no. 15, 2008, p. 7117.

6. (a) YANG, J., YANG, S., ZHOU, S., LU, D., JI, L., LI, Z., YU, S., MENG, X., Eur. J. Med. Chem., 122, 2016, p. 488. (b) HAN, F., WANG, P., ZHANG, W., LI, J., ZHANG, Q., QI, X., LIU, M., Biomed. Pharmacother., 80, 2016, p. 151. (c) BIERSACK, B., EFFENBERGER, K., KNAUER, S., OCKER, M., SCHOBERT, R., Eur. J. Med. Chem., 45, no. 11, 2010, p. 4890.

7. (a) ABHALE, Y. K., SASANE, A. V., CHAVAN, A. P., SHEKH, S. H., DESHMUKH, K. K., BHANSALI, S., NAWALE, L., SARKAR, D., MHASKE, P. C., Eur. J. Med. Chem., 132, 2017, p. 333. (b) MORASKI, G. C., MARKLEY, L. D., CHANG, M., CHO, S., FRANZBLAU, S. G., HWANG, C. H., BOSHOFF, H., MILLER, M. J., Bioorg. Med. Chem., 20, no. 7, 2012, p. 2214.

8. WHITE JR., R. L., WESSELS, F. L., SCHWAN, T. J., ELLIS, K. O., J. Med. Chem., 30, no. 2, 1987, p. 263.

9. (a) MATHEW, J. E., DIVYA, G., VACHALA, S. D., MATHEW, J. A., JEYAPRAKASH, R. S., J. Pharm. Res., 6, no. 1, 2013, p. 210. (b) STANKOVA, I., SPASOVA, M., Z. Naturforsch., 64c, no. 3-4, 2009, p. 176. 10. IKEMOTO, N., MILLER, R. A., FLEITZ, F. J., LIU, J., PETRILLO, D. E., LEONE, J. F., LAQUIDARA, J., MARCUNE, B., KARADY, S., ARMSTRONG, III, J. D., VOLANTE, R. P., Tetrahedron Lett., 46, no. 11, 2005, p. 1867.

11. (a) TILVI, S., SINGH, K. S., Curr. Org. Chem., 20, no. 8, 2016, p. 898. (b) JIN, Z., Nat. Prod. Rep., 33, no. 11, 2016, p. 1268. (c) DAVYT, D., SERRA, G., Mar. Drugs, 8, no. 11, 2010, p. 2755.

12. (a) DE CASTRO, P. P., CARPANEZ, A. G., AMARANTE, G. W., Chem. Eur. J., 22, no. 30, 2016, p. 10294. (b) FISK, J. S., MOSEY, R. A., TEPE, J. J., Chem. Soc. Rev., 36, no. 9, 2007, p. 1432.

13. SANDHAR, R. K., SHARMA, J. R., MANRAO, M. R., Indian J. Heterocycl. Chem., 13, no. 2, 2003, p. 119. 
14. (a) SUNEL, V., BASU, C., An. St. Univ. Al. I. Cuza lasi, Chimie, 7, no. 1, 1999, p. 111. (b) SUNEL, V., CIOVICA, S., ASANDEI, N., SOLDEA, C., Cellulose Chem. Tech., 29, no. 1, 1995, p. 11. (c) BUDEANU, C., IVAS, E., SUNEL, V., Rev. Chim. (Bucharest), 32, no. 5, 1981, p. 454.

15. (a) BALA, S., SAINI, M., KAMBOJ, S., Int. J. ChemTech Res., 3, no. 3, 2011, p. 1102. (b) PINTO, I. L., WEST, A., DEBOUCK, C. M., DILELLA, A. G., GORNIAK, J. G., O'DONNELL, K. C., O'SHANNESSY, D. J ., PATEL, A., JARVEST, R. L., Bioorg. \& Med. Chem. Lett., 6, no. 20, 1996, p. 2467.

16. (a) AHMAD, I., SHAGUFTA, Int. J. Pharm. Pharm. Sci., 7, no. 3, 2015,

p. 19. (b) WOZEL, V. E. G., Dermatol. Clinics, 28, no. 3, 2010, p. 599.

(c) CHO, S. C., RHIM, J. H., SON, Y. H., LEE, S. J., PARK, S. C., Exp. Mol. Med., 42, no. 3, 2010, p. 223.

17. BARBUCEANU, S.-F., BANCESCU, G., DRAGHICI, C., BARBUCEANU, F., CRETU, O. D., APOSTOL, T. V., BANCESCU, A., Rev. Chim. (Bucharest), 63, no. 4, 2012, p. 362.

18. BARBUCEANU, S.-F., SARAMET, G., BANCESCU, G., DRAGHICl, C., APOSTOL, T.-V., TARAN, L., DINU-PIRVU, C. E., Rev. Chim. (Bucharest), 64, no. 4, 2013, p. 355.

19. BARBUCEANU, S. F., SOCEA, L. I., DRAGHICI, C., PAHONTU, E. M., APOSTOL, T. V., BARBUCEANU, F., Rev. Chim. (Bucharest), 68, no. 10, 2017, p. 2436.

20. APOSTOL, T.-V., DRAGHICI, C., DINU, M., BARBUCEANU, S.-F., SOCEA, L. I., SARAMET, I., Rev. Chim. (Bucharest), 62, no. 2, 2011, p. 142.

21. APOSTOL, T.-V., SARAMET, I., DRAGHICl, C., BARBUCEANU, S.-F., SOCEA, L. I., ALMAJ AN, G. L., Rev. Chim. (Bucharest), 62, no. 5, 2011 , p. 486.
22. GUILHERMINO, L., DIAMANTINO, T., SILVA, M. C., SOARES, A. M. V. M., Ecotoxicol. Environ. Saf., 46, no. 3, 2000, p. 357.

23. SOCEA, L.-I., SOCEA, B., SARAMET, G., BARBUCEANU, S.-F., DRAGHICl, C., CONSTANTIN, V. D., OLARU, O. T., Rev. Chim. (Bucharest), 66, no. 8, 2015, p. 1122.

24. NITULESCU, G. M., IANCU, G., NITULESCU, G., IANCU, R. C., BOGDANICI, C., VASILE, D., Rev. Chim. (Bucharest), 68, no. 4, 2017, p. 754.

25. SOCEA, L. I., BARBUCEANU, S. F., SOCEA, B., DRAGHICI, C., APOSTOL, T. V., PAHONTU, E. M., OLARU, O. T., Rev. Chim. (Bucharest), 68, no. 11, 2017, p. 2503.

26. BARBUCEANU, S. F., OLARU, O. T., NITULESCU, G. M., DRAGHICl, C., SOCEA, L. I., BARBUCEANU, F., ENACHE, C., SARAMET, G., Rev. Chim. (Bucharest), 69, no. 9, 2018, p. 2346.

27. NITULESCU, G., NICORESCU, I. M., OLARU, 0. T., UNGURIANU, A., MIHAI, D. P., ZANFIRESCU, A., NITULESCU, G. M., MARGINA, D., Int. J. Mol. Sci., 18, no. 10, 2017, p. 2217.

28. SOCEA, L. I., BARBUCEANU, S. F., ISCRULESCU, L., SOCEA, B., HRUBARU, M., PAHONTU, E. M., DIACONU, C. C., BRATU, O. G., OLARU, 0. T., Rev. Chim. (Bucharest), 69, no. 12, 2018, p. 3341.

29. BARBUCEANU, S.-F., OLARU, O. T., SOCEA, L.-I., DRAGHICl, C., SARAMET, G., BARAITAREANU, S., SOCEA, B., BARBUCEANU, F., Rev. Chim. (Bucharest), 70, no. 1, 2019, p. 13.

30. MAVRODIN, A., ZOTTA, V., STROENESCU, V. M., OTELEANU, D., Pharm. Zentr. Dtschl., 95, no. 9, 1956, p. 353.

31. SCHIKETANZ, I., DRAGHICl, C., SARAMET, I., BALABAN, A. T., Arkivoc, (ii), 2002, p. 64.

32. WASSERMAN, H. H., VINICK, F. J., J. Org. Chem., 38, no. 13, 1973, p. 2407.

$\overline{\text { Manuscript received: } 10.05 .2018}$ 